\title{
Mindfulness-based online intervention on mental health and quality of life among COVID-19 patients in China: an intervention design
}

Ming-Yu Si ${ }^{1 \dagger}$, Wei-Jun Xiao ${ }^{1 \dagger}$, Chen Pan², Hao Wang ${ }^{1}$, Yi-Man Huang ${ }^{1}$, Jun Lian ${ }^{1}$, Winnie W. S. Mak ${ }^{3}$, Zhi-Wei Leng ${ }^{1}$, Xiao-You Su ${ }^{1 *} \mathbb{0}$, Qiu-Ping Tang ${ }^{2^{*}}$, Yu Jiang ${ }^{1}$, Lu-Zhao Feng ${ }^{1 *}$, Wei-Zhong Yang ${ }^{1}$ and Chen Wang ${ }^{1,4,5}$

\begin{abstract}
Background: COVID-19 can lead to increased psychological symptoms such as post-traumatic stress disorder (PTSD), depression, and anxiety among patients with COVID-19. Based on the previous mindfulness-based interventions proved to be effective, this protocol reports a design of a randomized controlled trial aiming to explore the efficacy and possible mechanism of a mindful living with challenge (MLWC) intervention developed for COVID-19 survivors in alleviating their psychological problems caused by both the disease and the pandemic.

Methods: In April 2021, more than 1600 eligible participants from Hubei Province of China will be assigned 1:1 to an online MLWC intervention group or a waitlist control group. All participants will be asked to complete online questionnaires at baseline, post-program, and 3-month follow-up. The differences of mental health status (e.g. PTSD) and physical symptoms including fatigue and sleeplessness between the COVID-19 survivors who receiving the online MLWC intervention and the control group will be assessed. In addition, the possible mediators and moderators of the link between the MLWC intervention and target outcomes will be evaluated by related verified scales, such as the Five Facets Mindfulness Questionnaire. Data will be analyzed based on an intention-to-treat approach, and SPSS software will be used to perform statistical analysis.
\end{abstract}

Discussion: The efficacy and potential mechanism of MLWC intervention in improving the quality of life and psychological status of COVID-19 survivors in China are expected to be reported. Findings from this study will shed light on a novel and feasible model in improving the psychological well-being of people during such public health emergencies.

\footnotetext{
*Correspondence: suxiaoyou@hotmail.com; tt96@sina.com;

fengluzhao@cams.cn

${ }^{\dagger}$ Ming-Yu Si and Wei-Jun Xiao joint first authors

1 School of Population Medicine and Public Health, Chinese

Academy of Medical Sciences and Peking Union Medical College, 9

DongDanSanTiao, Dongcheng, Beijing, China

${ }^{2}$ Department of Clinical Psychology, The Third Xiangya Hospital of Central

South University, No. 138 Tongzipo Road, Yuelu, Changsha, Hunan, China

Full list of author information is available at the end of the article

${ }^{\dagger}$ Xiao-You Su, Qiu-Ping Tang and Lu-Zhao Feng co-corresponding authors
}

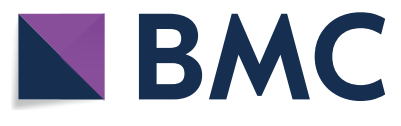

The Author(s) 2021. Open Access This article is licensed under a Creative Commons Attribution 4.0 International License, which. permits use, sharing, adaptation, distribution and reproduction in any medium or format, as long as you give appropriate credit to the original author(s) and the source, provide a link to the Creative Commons licence, and indicate if changes were made. The images or other third party material in this article are included in the article's Creative Commons licence, unless indicated otherwise in a credit line to the material. If material is not included in the article's Creative Commons licence and your intended use is not permitted by statutory regulation or exceeds the permitted use, you will need to obtain permission directly from the copyright holder. To view a copy of this licence, visit http://creativecommons.org/licenses/by/4.0/. The Creative Commons Public Domain Dedication waiver (http://creativeco mmons.org/publicdomain/zero/1.0/) applies to the data made available in this article, unless otherwise stated in a credit line to the data. 
Trial registration Chinese Clinical Trial Registry (ChiCTR), ChiCTR2000037524; Registered on August 29, 2020, http:// www.chictr.org.cn/showproj.aspx?proj=60034.

Keywords: COVID-19, Internet, Mindfulness-based intervention, Mental health, Randomized controlled trial, China

\section{Background}

Coronavirus disease 2019 (COVID-19), the infection caused by severe acute respiratory syndrome coronavirus 2 (SARS-CoV-2), was first reported on December 31, 2019. On March 11, 2020, the World Health Organization (WHO) declared the COVID-19 as a pandemic due to its rapid global spreading. Globally, there have been 122536880 COVID-19 cases and 2703780 deaths, particularly among vulnerable populations such as patients with chronic diseases and the elderly $[1,2]$. As of 24:00 pm on March 22, 2021, China alone has resulted in 102566 cases and 4850 deaths due to COVID-19 [3]. It can be seen that the mortality rate of COVID-19 has been declining recently, which means that more and more patients have been released from the hospital after being cured. Therefore, the focus has now been shifted to taking measures to rehabilitate COVID-19 survivors, including the restoration of their physical functions and psychological development.

Evidence suggests that this disease has psychological consequences on healthcare workers, students, psychiatric patients and general workers, especially for COVID19 patients [4-13]. Immediately after the outbreak, a considerable proportion of patients with COVID-19 reported post-traumatic stress disorder (PTSD) (more than 33.0\%), depression (60.2\%), and anxiety (55.3\%) symptoms in Shanghai, China [9]. In addition, a survey conducted in March, 2020 showed that $45.9 \%$ of patients had symptoms of depression, $38.8 \%$ had anxiety and $54.1 \%$ had insomnia in Wuhan, China [10]. COVID-19 survivors in other countries have similar psychological status [11-13]. In Ecuador, one of the most affected countries by the COVID-19 pandemic in Latin America, of the 759 confirmed or suspected COVID-19 patients, $20.3 \%$ showed moderate to severe depressive symptoms, while $22.5 \%$ showed moderate to severe anxiety [11]. In addition, $22.6 \%$ and $16.3 \%$ of respondents reported clinically concerning levels of psychological impact in Vietnam and the Philippines, the Asian countries with similar cultural context as China $[12,13]$. Over time, while the mental symptoms and quality of life of COVID-19 survivors might improve in a certain extent, they are still significantly worse when compared to the rest of the population who did not suffer from COVID-19. Therefore, the psychological impacts of COVID-19 should not be overlooked and should be investigated from the longterm public health perspectives.
In view of the current epidemic situation and the corresponding prevention and control requirements, internet-based interventions can provide an alternative to face-to-face therapy in enhancing mental health and quality of life for patients with COVID-19. Internetbased healthcare has enabled patients with a variety of geographical distance to receive the same therapy and get benefits from the same interventions, such as digital cognitive behavioural therapy for insomnia [14-16]. This form of psychotherapy can provide treatment and intervention efficiently to patients under the requirements of social distancing during the COVID-19 epidemic [14]. Mindfulness-based intervention (MBI) is an approach that focuses on the cultivation of conscious awareness, and orientation towards the present moment with curiosity and openness, which has been shown helpful in the treatment of mental health [17-20]. Unlike behavioral therapy, which involves relaxation techniques and changing the schedule of daily activities, mindfulnessbased interventions help patients cope with anxiety and depression by enhancing their ability to manage stress and reducing negative coping behaviors, hence are more adaptive and persistent [20]. Especially when combined with the web-based technology, mindfulness-based intervention can benefit patients who are infected and treated in isolation wards, and those who are quarantined with no access to psychotherapy as well. $[17,20]$ An internet-based integrated intervention including some mindfulness skills on depression and anxiety symptoms in Chinese patients with COVID-19 has demonstrated that patients of the intervention group exhibited significantly decreased levels of depression and anxiety symptoms in comparison with those of the control group over a 2-week study period [18].

In the mainland of China, WeChat App and its small programs have dominated the browsing time of mobile phone users [21] Given the emerging need for psychological rehabilitation among COVID-19 survivors recently, this randomized controlled trial will be conducted in this population to examine the effectiveness and feasibility of a 6-week Mindful Living With Challenge (MLWC) intervention focusing on mental health and quality of life via WeChat. We hypothesized that the training could enhance the physical and mental health at post-program and 3-month follow-up. 


\section{Methods/design}

\section{Study design}

This is a prospective, randomized, parallel-group, double-blind, blank controlled trial involving an online 6-week MLWC intervention with a 3-month follow-up. More than 1600 COVID-19 survivors over 18 years old will be randomized to: (1) intervention group: this group will receive a baseline questionnaire followed by a 6-week web-based MLWC intervention, and two questionnaire surveys at post-program and 3 months after the intervention, or (2) waitlist control group: participants in this group will receive three questionnaire surveys conducted simultaneously with the intervention group, and start the same intervention after a 3-month waitlist period (Fig. 1). This study protocol is reported in accordance with SPIRIT reporting guidelines [22]

\section{Study objectives}

The primary study objective of this study is to assess the efficacy of the MLWC intervention on PTSD, depression, and anxiety among COVID-19 survivors. The secondary study objective is to investigate the effects of the MLWC

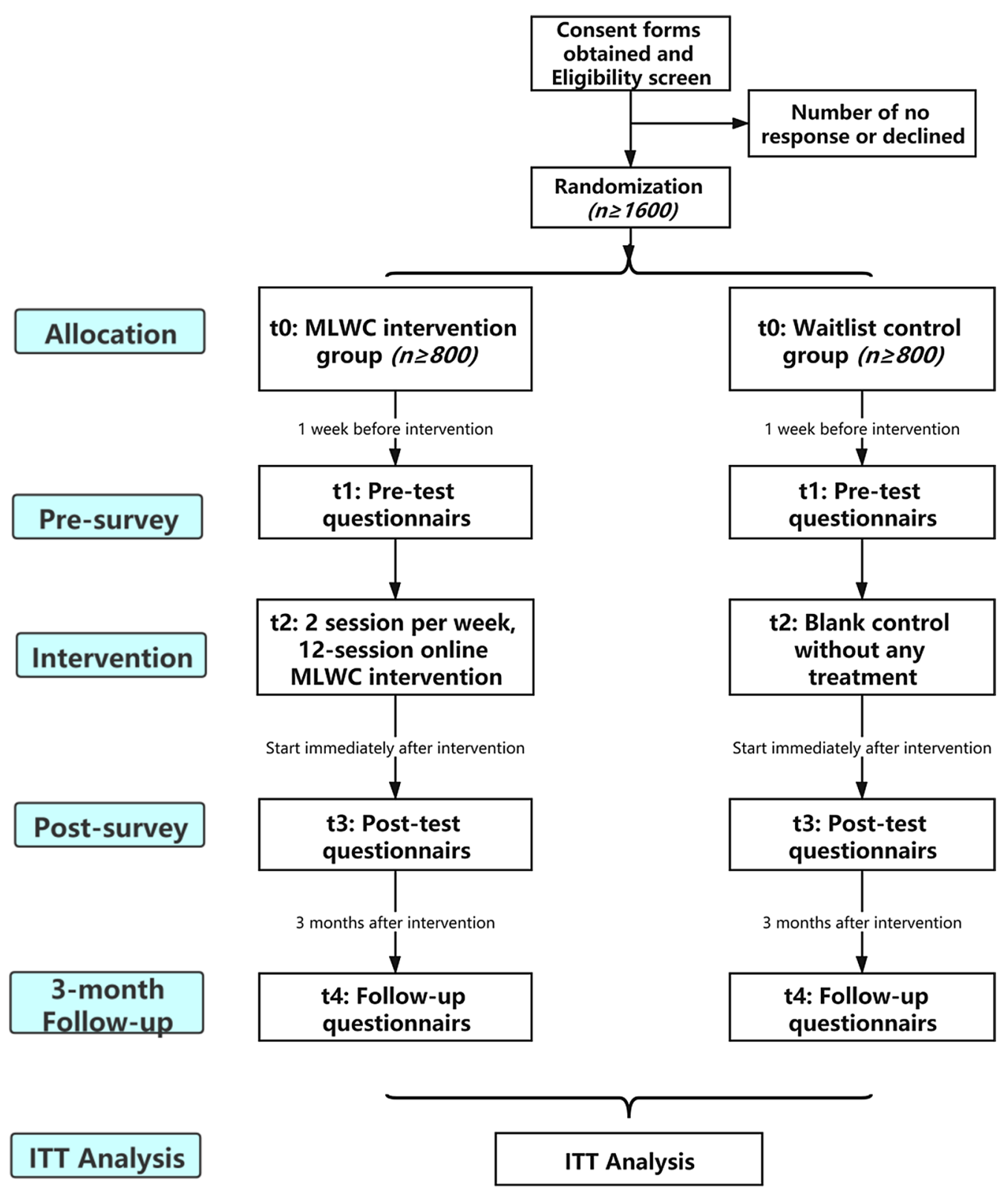

Fig. 1 Flow chart of the protocol. MLWC: Mindful living with challenge; ITT: Intention-To-Treat 
intervention on fatigue and sleep quality among COVID19 survivors. The exploratory objective is to determine how mindfulness training affects mental health and quality of life among COVID-19 survivors and to evaluate the possible mediators and moderators (mindfulness, stillness, nonattachment, resilience and perceived social support) of the link between the MLWC intervention and target outcomes.

\section{Settings}

The study will be carried out at the Hubei Provincial Hospital of Integrated Traditional Chinese and Western Medicine, Hubei Province, China. All researchers and support staff in this project will be trained based on the same protocol and are required to have an educational background in medicine or public health. Digital informed consent will be obtained from all subjects to ensure their voluntary participation. This study is planned to be performed in April 2021 and will last for about 4 months (The study has not been carried out before the submission).

\section{Sample size}

A priori power analysis has been conducted on the statistical software G*Power 3.1.9.2 (Heinrich-HeineUniversität Düsseldorf). The trial between the MLWC intervention group and the control group will be tested superiority in promoting mental health status at a superiority margin of $10 \%$. Thus, with a 1:1 allocation to each group and allowing for a $40 \%$ attrition rate, at least 1600 participants will be recruited (power $=0.9, \alpha=0.05$, two-tail).

\section{Recruitment}

Participants will be recruited through advertisements posted in hospitals and publicity by doctors in outpatient clinics or patient contact groups. Interested participants will be informed about the benefits and possible risks of participation in the study, including failure to achieve the expected intervention effects. Completion of electronic informed consent will be required before screening the eligibility of participating in the study.

Once the informed consent is obtained, a trained research staff will complete the eligibility checklists and

Table 1 Schedule of enrolment, interventions, and assessments

\begin{tabular}{|c|c|c|c|c|c|c|}
\hline \multicolumn{7}{|l|}{ Study period } \\
\hline \multirow[b]{2}{*}{ Time point } & \multirow{2}{*}{$\begin{array}{l}\text { Enrolment } \\
0\end{array}$} & \multirow{2}{*}{$\begin{array}{l}\text { Allocation } \\
t_{0}\end{array}$} & \multicolumn{3}{|c|}{ Post-allocation } & \multirow{2}{*}{$\begin{array}{l}\text { Close-out } \\
\mathbf{t}_{4 \text { Follow-up }} \\
\text { assessment }\end{array}$} \\
\hline & & & $t_{1 \text { baseline }}$ & $t_{2 \text { Intervention period }}$ & $t_{3 \text { Post-assessment }}$ & \\
\hline \multicolumn{7}{|l|}{ Enrolment } \\
\hline Eligibility screen & $x$ & & & & & \\
\hline Informed consent & $x$ & & & & & \\
\hline Allocation & & $x$ & & & & \\
\hline \multicolumn{7}{|l|}{ Interventions } \\
\hline The Mindful Living with Challenge intervention group & & & & $x$ & & \\
\hline Waitlist control group & & & & $x$ & & \\
\hline \multicolumn{7}{|l|}{ Assessments } \\
\hline \multicolumn{7}{|l|}{ Primary outcome measures } \\
\hline Impact of Events Scale-Revised & & & $x$ & & $x$ & $x$ \\
\hline Patient Health Questionnaire & & & $x$ & & $x$ & $x$ \\
\hline Generalized Anxiety Disorder Questionnaire & & & $x$ & & $x$ & $x$ \\
\hline Peace of Mind Scale & & & $x$ & & $x$ & $x$ \\
\hline \multicolumn{7}{|l|}{ Secondary outcome measures } \\
\hline Fatigue Scale-14 & & & $x$ & & $x$ & $x$ \\
\hline Pittsburgh Sleep Quality Index & & & $x$ & & $x$ & $x$ \\
\hline \multicolumn{7}{|l|}{ The possible mediators and moderators } \\
\hline Five Facets Mindfulness Questionnaire & & & $x$ & & $x$ & $x$ \\
\hline The Nonattachment Scale & & & $x$ & & $x$ & $x$ \\
\hline The Stillness Scale & & & $x$ & & $x$ & $x$ \\
\hline The Resilience Style Questionnaire & & & $x$ & & $x$ & $x$ \\
\hline The Social Support Scale & & & $x$ & & $x$ & $x$ \\
\hline
\end{tabular}

' $X$ ' represents the research procedure or content involved in each time point 
record the candidates who fail to meet the inclusion criteria. Furthermore, a senior nurse will record the mobile phone number of participants to facilitate follow-up services. All of the above personal information will be kept confidential on an encrypted laptop and used for research purposes only. Table 1 shows schedule of enrolment, interventions and assessments.

\section{Eligibility criteria \\ Inclusion criteria}

Participants must first agree to the electronic informed consent in order to participate in the study as required by the ethics committee. The participants have to meet the following criteria:

1) Over 18 and under 65 years old

2) Have a history of COVID-19 infection

3) Proficiency in Chinese

4) Be able to independently cooperate with doctors to complete various scale assessments

5) Have a mobile communication equipment such as a mobile phone, and a WeChat account.

6) Mobile equipment can access the internet at any time

7) Have not received medications for PTSD, depression, anxiety, fatigue, or sleep disorders within 1 month prior to enrollment in the study.

\section{Exclusion criteria}

Those who meet any of the below criteria will be excluded:

1) Have serious cognitive impairment.

2) Have serious heart, brain, lung, kidney, liver, and other medical diseases or tumors.

3) Difficult to cooperate with the questionnaire survey and intervention.

\section{Randomization and blinding}

Participants who meet the eligibility criteria will be randomly assigned (in a 1:1 ratio) to the online MLWC intervention group or waitlist control group. They are supposed to provide informed consent by clicking the 'I agree to join in the research' button on the electronic details page of the study. After that, a Quick Response (QR) code will pop up on the page. By scanning this code, participants will be randomly assigned to the intervention or control group (in a 1:1 ratio) by computer-generated numbers. The trial will blind all people who might influence the outcome assessments, including the participants, data analysts, and outcome assessors. Before the trial, the principal investigator of the project will organize training on the randomization procedure and ensure that each research staff is clearly aware of their responsibilities.

\section{Intervention}

\section{The mindful living with challenge (MLWC) intervention}

Participants in the intervention group are required to follow the developed WeChat small program by scanning the QR code, and then receive the online MLWC intervention. The MLWC intervention will be developed by two psychiatrists, and one of them has been certified in the Training of Mindfulness Facilitation program at the Mindful Awareness Research Center of University of California, Los Angeles (UCLA). The MLWC intervention adopts some elements from mindfulness-based stress reduction and Mindful Awareness Practices, and includes mindfulness meditation and mindful stretching as core components. A similar intervention has been successfully used on attention monitoring and acceptance in pregnant women in China [19]. Through a series of mindfulness training courses, participants are hypothesized to increase their ability to regulate their emotions and learn to observe the physical pain and psychological frustration caused by COVID-19 infection with equanimity. The purpose of the study is to help participants improve their skills in coping with psychological symptoms, improve their quality of life during recovery, and prepare to return to a normal life.

The MLWC intervention will involve internet-based mindfulness training conducted in six weekly courses of two sessions a week and $0.5 \mathrm{~h}$ per session, which focus on the four important parts of MBI: attention control, selfawareness, acceptance and discernment, and relating to difficult thoughts/emotions. Two new sessions of online lessons will be updated weekly in six weeks. The study participants are expected to spend an average of $1 \mathrm{~h}$ per week viewing each lesson, which consisted of a theoretical instrument, some meditation practices, and homework in the form of audio and video. Participants will be required to perform formal/informal mindfulness practice at home for $5 \mathrm{~min}$ daily initially, advancing to $20 \mathrm{~min}$ daily at the end of the intervention period, and to record or report their difficulties and progress during the practice. To enhance adherence, push notifications from the WeChat small program will be sent to participants daily to remind them about their weekly attendance and regular practice. See Table 2 for the context of the MLWC intervention. 


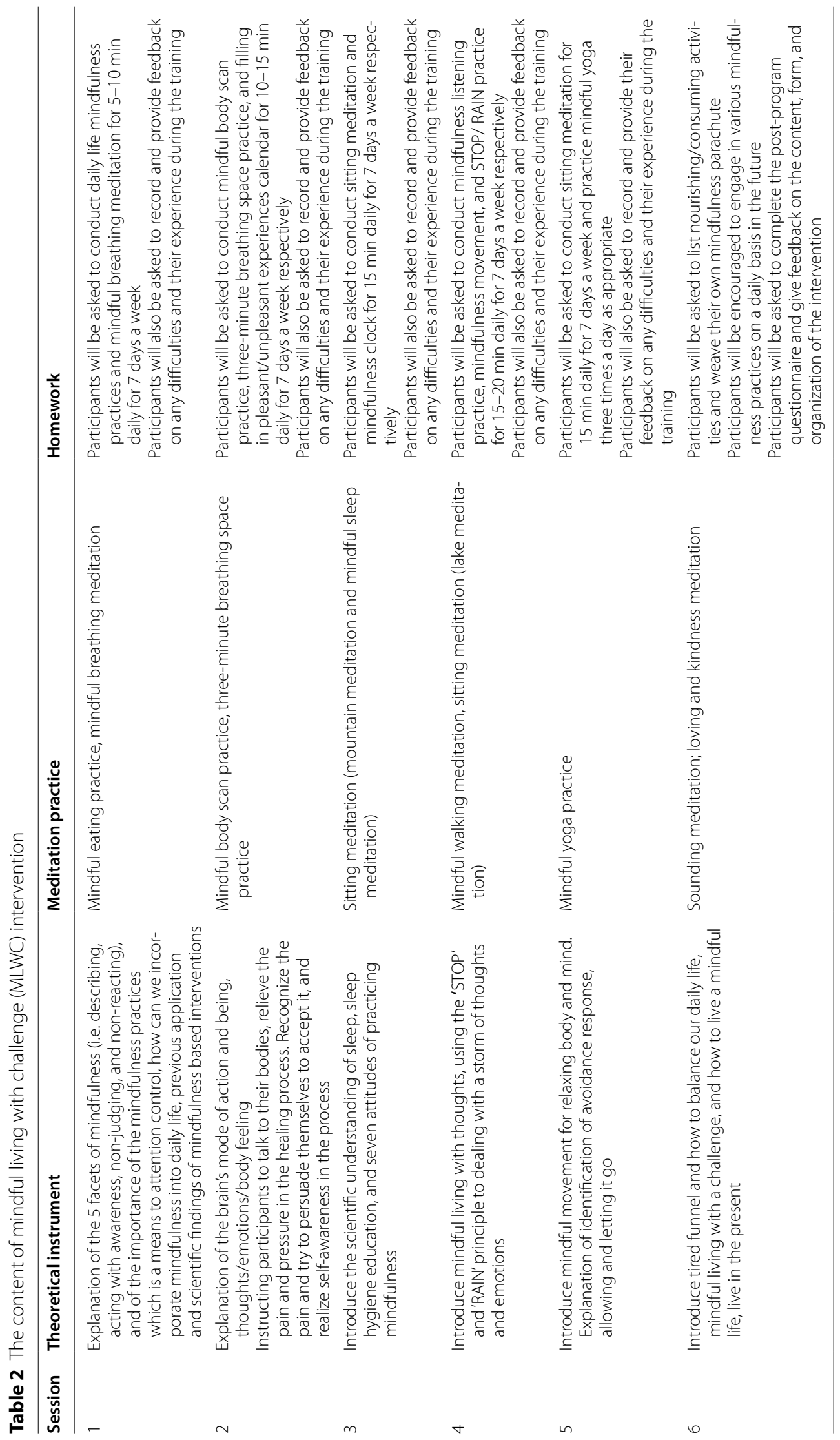




\section{Control group}

Participants in the waitlist control group will receive the full 12-session online MLWC intervention upon completion of all treatment procedures by the intervention group after the 3-month follow-up survey.

\section{Measures}

\section{Adherence}

Participants will be informed of the importance of attending each session and required to complete the homework, as the effectiveness of the MLWC intervention on mental health and quality of life is dependent on adherence. Adherence to the intervention was examined through documentation of completed sessions. Firstly, the internet-based intervention platform can automatically record which participant has logged into each session and how much time they spend on mindfulness training per week. In addition, participants will be reminded to complete their homework after completion of each session. Moreover, the post-program questionnaire will be sent to the participants at the end of the intervention to ask about their adherence issues.

\section{Outcome measurements}

All scales to be used in this study are validated Chinese version.

\section{Primary outcome: PTSD, depression, anxiety and peace of mind}

The psychological impact of COVID-19 will be assessed by the 22-item Impact of Event Scale-Revised (IES-R). IES-R is composed of three subscales and aims to measure the mean avoidance, intrusion, and hyperarousal (Cronbach's $\alpha=0.80$ ) [23-25]. Depression symptoms will be assessed by the 9-item Patient Health Questionnaire (PHQ-9) (Cronbach's $\alpha=0.86$ ) [26-28]. The level of general anxiety will be assessed by the 7-item Generalized Anxiety Disorder Questionnaire (Cronbach's $\alpha=0.89$ ) $[29,30]$. Additionally, the 7-item Peace of Mind Scale developed by Lee et al. will be used to measure the peace of mind, one part of the well-being in the Chinese culture (Cronbach's $\alpha=0.90)[31,32]$.

\section{Secondary outcomes: fatigue and sleep quality}

Fatigue scale-14 (FS-14) assesses the patient's level of physical fatigue and mental fatigue (Cronbach's $\alpha=0.81$ ) [33, 34]. The 19-item Pittsburgh Sleep Quality Index (PSQI) assesses respondents' sleep disturbances during the past month (Cronbach's $\alpha=0.84$ ). [35, 36] PSQI can be divided into seven component scores: subjective sleep quality, sleep latency, sleep duration, habitual sleep efficiency, sleep disturbances, use of medication, and daytime dysfunction [35].

\section{The possible mediators and moderators: mindfulness, nonattachmet, stillness, resilience and perceived social support}

The mindfulness level will be assessed by the 20-item short formed Five Facets of Mindfulness Questionnaire (FFMQ-SF). FFMQ-SF is a self-reported questionnaire with five mindfulness domains: observing, describing, acting with awareness, non-judgment, and non-reactivity (Cronbach's $\alpha=0.86)[37,38]$. Nonattachment will be evaluated by the 7-Item Nonattachment Scale (NAS-7) (Cronbach's $\alpha=0.82$ ) [39-41]. To measure the extent to which participants experience stillness, the 11-item Stillness Scale will be used. It is developed and validated by one of the authors with three subscales: tranquility, concentration, and equanimity. (Cronbach's $\alpha$ ranges from 0.72 to 0.75 ). In addition, participants' resilience will be measured by the 17-item Resilience Style Questionnaire (RSQ) (Cronbach's $\alpha=0.88$ ) [42]. The level of emotional and material social support that participants receive will be measured with two items used in previous studies (Cronbach's $\alpha=0.68$ ) [43].

\section{Data collection, management, and monitoring}

Data will be collected at baseline (one week before the intervention), post-intervention (one week after the last session of MLWC), and at 3-month of follow-up. The questionnaire comprises structured and open-ended questions about mental health status, quality of life, and satisfaction with the program. Demographics will be collected at baseline, including age, employment status, education level, marital status, and other information.

Electronic questionnaires powered by the REDCap software will be used to collect data, hence there will be no formal data monitoring committee. Survey questions filled up by the participants will be automatically uploaded to the read-only web-based database to ensure the authenticity of the data. Moreover, our researchers will promptly check the collected data after each questionnaire survey.

\section{Statistical analysis}

IMB SPSS Statistics 22.0 (IBM Corp., Armonk, NY, USA) will be used to process data and conduct a $t$-test, chi-square test, and correlation analysis. Analyses will be conducted based on an intention-to-treat approach, and two-sided with $P<0.05$ will be considered statistically significant. Quantitative data accords with normal distribution will be described as mean \pm standard deviation $(S D)$, and qualitative data will be expressed as the 
number (percentage). Student's $t$-test and the chi-square test will be employed to examine between-group differences in the baseline. The repeated measures ANOVA will be used to compare the scores for the six instruments, and the paired-samples $t$-test will be used to test for within-group differences. We will also conduct a correlation analysis to test the associations between variables in pre-program and post-program in each group. Structural Equation Model will be conducted to explore the interaction of the variables.

Furthermore, in order to elaborate on the meaning of the relationship between the mindfulness training and the outcome variables of mental state and quality of life among COVID-19 survivors, an analysis of mediator or moderator effects will be conducted and some more indepth information about the research may be explained. We will use the hierarchical multiple regression analyses to test how or why some facets of mindfulness may take a role in our outcomes.

\section{Discussion}

With the unprecedented global health challenge the COVID-19 pandemic has created, the secondary crisis of mental health problems occurred with the outbreak cannot be ignored in a very long period of time. As of March 22, 2021, there are 97716 COVID-19 survivors cured and discharged from hospital in China, about one-third of them (i.e. 32572 patients) may be suffering from mental illness as reported by the survey results in Wuhan, China $[3,10]$. Prolonged exposure to stressful hospitalization, complex medical examination procedures, and worries about the difficulty of returning to normal life can lead to long-term psychological harm [44]. This will undoubtedly affect the normal operation of many families and bring huge health and economic pressure to the country. Therefore, appropriate innovative mental health support with social distancing merit is warranted to be implemented during the COVID-19 pandemic and its aftermath.

This article describes the design and content of a parallel-group randomized controlled trial (RCT). Our study is the first large sample size study to explore the effect and underlying mechanism of the online MLWC intervention aiming to improve mental health and quality of life among COVID-19 survivors in China. If the study demonstrates that the web-based MLWC is efficacious in such improvement, this user-friendly and convenient mode of mental health intervention would be valuable to treat psychological symptoms and improve their quality of life among COVID-19 rehabilitation patients. The intervention materials also have the potential to be utilized in other populations in various similar situations.
Although previous studies showed that face-to face mindfulness practices are effective to alleviate mental and physical symptoms including depression and sleeplessness $[17-19,45]$, the web-based mindfulness intervention were proved to have potential in improving the mental health of various study population with more feasibility and acceptability [46]. Currently, the WeChat App and its small programs have become an integral part of everyday life for the majority of the population in China [21]. To ensure the efficacy and the quality of the mindfulness intervention, the content of the intervention course will be developed by a team with clinical psychology background and delivered by a psychiatrist with mindfulness training qualification. Also, to enhance the adherence of the mindfulness-based intervention, professionals in information technology will design the specific small program for managing the questionnaire collection and distribution of the embedded video and audio courses during the study period.

The evaluation of the online MLWC intervention proposed in this study for the treatment of psychological symptoms among COVID-19 survivors is scarce in the international literature. The current study will use RCT, double-blind, evidence-based meditation design, and a longer period of intervention and follow-up, which may ensure more solid conclusions compared to similar studies [18, 47]. Moreover, since some studies have found that the mindfulness intervention did not affect all of the mindfulness facets $[48,49]$, we will explore 12 sessions of the MLWC intervention as intermediaries on the outcome measures, to determine which sessions have the greatest impact on the outcome, and provide more solid evidence for future psychological interventions. Furthermore, given the inexpensive and portable features of the online MLWC intervention, it can be more easily implemented in harder-to-reach but internet-accessible populations. However, it should be noted that, this study will mainly use questionnaires to measure psychiatric symptoms and do not make clinical diagnosis through structured clinical interview and functional neuroimaging, which may result in measurements that are inconsistent with clinical examinations $[50,51]$.

\footnotetext{
Abbreviations

COVID-19: Coronavirus disease 2019; MLWC: Mindful living with challenge; MBI: Mindfulness-based intervention; PTSD: Post-traumatic stress disorder; RCT : Randomized controlled trial; CONSORT: Consolidated Standards of Reporting Trials; IES-R: Impact of Events Scale-Revised; PHQ-9: Patient Health Questionnaire; GAD-7: Generalized Anxiety Disorder Questionnaire; PoM: Peace of Mind Scale; FS-14: Fatigue Scale-14; PSQI: Pittsburgh Sleep Quality Index; FFMQ: Five Facets Mindfulness Questionnaire; NAS-7: The Nonattachment Scale; RSQ: The Resilience Style Questionnaire; SD: Standard deviation; P: Probability.
} 


\section{Acknowledgements \\ Not applicable.}

\section{Authors' contributions}

X-YS, M-YS and W-JX prepared the first draft. Q-PT and CP provided overall guidance for the mindfulness-based interventions. HW, Y-MH and JL were responsible for the questionnaire design. WWSM provided instrumental support for the part of outcome measures. CW and Z-WL will be responsible for the overall project in Wuhan, China. L-ZF, W-ZY and YJ finalized the manuscript based on the comments from other authors. All authors read and approved the final manuscript.

\section{Funding}

The Innovative Engineering Program sponsored by the Chinese Academy of Medical Sciences (2020-12M-2-015, 2019-12M-2-005) and Asian Regional Special Cooperation Fund of National Health Commission of the People's Republic of China (BLXM01)

\section{Availability of data and materials}

The original data generated from this study and the analyzed results will be available from the corresponding author upon reasonable request.

\section{Declarations}

\section{Ethics approval and consent to participate}

This study has been approved by the Ethical Review Committee of Institute of Pathogen Biology, Chinese Academy of Medical Sciences, Beijing, China (IPB2020-22). Electronic informed consents will be obtained from all participants prior to the baseline survey. Trial results and findings will be published in peerreviewed journals and scientific conferences.

\section{Consent for publication}

Not applicable.

\section{Competing interests \\ None.}

\section{Protocol amendments}

Any modifications to the protocol which may impact on the conduct of the study will be approved by the Ethical Review Committee of Institute of Pathogen Biology prior to implementation. Other minor amendments will just be agreed upon by the cooperation team and documented in a memorandum.

\section{Author details}

${ }^{1}$ School of Population Medicine and Public Health, Chinese Academy of Medical Sciences and Peking Union Medical College, 9 DongDanSanTiao, Dongcheng, Beijing, China. ${ }^{2}$ Department of Clinical Psychology, The Third Xiangya Hospital of Central South University, No. 138 Tongzipo Road, Yuelu, Changsha, Hunan, China. ${ }^{3}$ Diversity and Well-Being Laboratory, Department of Psychology, The Chinese University of Hong Kong, Shatin, NT, Hong Kong, China. ${ }^{4}$ Chinese Academy of Medical Sciences and Peking Union Medical College, Beijing, China. ${ }^{5}$ National Clinical Research Center for Respiratory Diseases, Beijing, China.

Received: 23 November 2020 Accepted: 3 April 2021

Published online: 17 May 2021

\section{References}

1. WHO. COVID-19 weekly epidemiological update, 21 March 2021. https:// www.who.int/publications/m/item/weekly-epidemiological-update-oncovid-19---23-march-2021. Accessed 22 Mar 2021.

2. World Health Organization. 2020. Coronavirus disease 2019 (COVID-19): Situation report 63. https://www.who.int/docs/default-source/coronaviru se/situation-reports/20200323-sitrep-63-Covid-19.pdf?sfvrsn $=$ d97 cb 6dd_2. Accessed 18 Nov 2020.

3. National Health Commission of the People's Republic of China. March 23: Daily briefing on novel coronavirus cases in China. http://en.nhc.gov.cn/ 2021-03/23/c_83286.htm. Accessed 22 Mar 2021.
4. Luo M, Guo L, Yu M, Jiang W, Wang H. The psychological and mental impact of coronavirus disease 2019 (COVID-19) on medical staff and general public - a systematic review and meta-analysis. Psychiatry Res. 2020;291:113190. https://doi.org/10.1016/j.psychres.2020.113190.

5. Si MY, Su XY, Jiang Y, Wang WJ, Gu XF, Ma L, et al. Psychological impact of COVID-19 on medical care workers in China. Infect Dis Poverty. 2020;9(1):113. https://doi.org/10.1186/s40249-020-00724-0.

6. Xie L, Luo H, Li M, Ge W, Xing B, Miao Q. The immediate psychological effects of Coronavirus Disease 2019 on medical and non-medical students in China. Int J Public Health. 2020;65(8):1445-53. https://doi.org/ 10.1007/s00038-020-01475-3.

7. Hao F, Tan W, Jiang L, Zhang L, Zhao X, Zou Y, et al. Do psychiatric patients experience more psychiatric symptoms during COVID-19 pandemic and lockdown? A case-control study with service and research implications for immunopsychiatry. Brain Behav Immun. 2020;87:100-6. https://doi. org/10.1016/j.bbi.2020.04.069.

8. Tan W, Hao F, Mclntyre RS, Jiang L, Jiang X, Zhang $L$, et al. Is returning to work during the COVID-19 pandemic stressful? A study on immediate mental health status and psychoneuroimmunity prevention measures of Chinese workforce. Brain Behav Immun. 2020;87:84-92. https://doi.org/ 10.1016/j.bbi.2020.04.055

9. Guo Q, Zheng Y, Shi J, Wang J, Li G, Li C, et al. Immediate psychological distress in quarantined patients with COVID-19 and its association with peripheral inflammation: a mixed-method study. Brain Behav Immun. 2020;88:17-27. https://doi.org/10.1016/j.bbi.2020.05.038.

10. Hu Y, Chen Y, Zheng Y, You C, Tan J, Hu L, et al. Factors related to mental health of inpatients with COVID-19 in Wuhan, China. Brain Behav Immun. 2020. https://doi.org/10.1016/j.bbi.2020.07.016.

11. Paz C, Mascialino G, Adana-Díaz L, Rodríguez-Lorenzana A, SimbañaRivera K, Gómez-Barreno L, et al. Behavioral and sociodemographic predictors of anxiety and depression in patients under epidemiological surveillance for COVID-19 in Ecuador. PLoS ONE. 2020;15(9):e0240008. https://doi.org/10.1371/journal.pone.0240008 (PMID: 32997705).

12. Le HT, Lai AJX, Sun J, Hoang MT, Vu LG, Pham HQ, et al. Anxiety and depression among people under the nationwide partial lockdown in Vietnam. Front Public Health. 2020;29(8):589359. https://doi.org/10.3389/ fpubh.2020.589359.

13. Tee ML, Tee CA, Anlacan JP, Aligam KJG, Reyes PWC, Kuruchittham V, et al. Psychological impact of COVID-19 pandemic in the Philippines. J Affect Disord. 2020;1(277):379-91. https://doi.org/10.1016/j.jad.2020.08.043.

14. Tran BX, Hoang MT, Vo LH, Le HT, Nguyen TH, Vu GT, et al. Telemedicine in the COVID-19 pandemic: motivations for integrated, interconnected, and community-based health delivery in resource-scarce settings? Front Psychiatry. 2020;11(11):564452. https://doi.org/10.3389/fpsyt.2020.564452.

15. Zhang MW, Ho RC. Moodle: the cost effective solution for internet cognitive behavioral therapy (I-CBT) interventions. Technol Health Care. 2017;25(1):163-5. https://doi.org/10.3233/THC-161261.

16. Soh HL, Ho RC, Ho CS, Tam WW. Efficacy of digital cognitive behavioural therapy for insomnia: a meta-analysis of randomised controlled trials. Sleep Med. 2020;75:315-25. https://doi.org/10.1016/j.sleep.2020.08.020.

17. Grossman P, Niemann L, Schmidt S, Walach H. Mindfulness-based stress reduction and health benefits. A meta-analysis. J Psychosom Res. 2004;57(1):35-43. https://doi.org/10.1016/S0022-3999(03)00573-7.

18. Wei N, Huang BC, Lu SJ, Hu JB, Zhou XY, Hu CC, et al. Efficacy of internetbased integrated intervention on depression and anxiety symptoms in patients with Covid-19. J Zhejiang Univ Sci B. 2020;21(5):400-4. https:// doi.org/10.1631/jzus.B2010013.

19. Yang M, Jia G, Sun S, Ye C, Zhang R, Yu X. Effects of an online mindfulness intervention focusing on attention monitoring and acceptance in pregnant women: a randomized controlled trial. J Midwifery Womens Health. 2019;64(1):68-77. https://doi.org/10.1111/jmwh.12944.

20. Ho CS, Chee CY, Ho RC. Mental health strategies to combat the psychological impact of COVID-19 beyond paranoia and panic. Ann Acad Med Singap. 2020;49(3):155-60.

21. Tencent. Tencent announces 2018 first quarter results. https://www.tence nt.com/en-us/articles/15000691526464720.pdf. Accessed 18 Nov 2020.

22. Chan AW, Tetzlaff JM, Gøtzsche PC, Altman DG, Mann H, Berlin JA, et al. SPIRIT 2013 explanation and elaboration: guidance for protocols of clinical trials. BMJ. 2013;346:e7586. https://doi.org/10.1136/bmj.e7586. 
23. Creamer M, Bell R, Failla S. Psychometric properties of the Impact of event scale-revised. Behav Res Ther. 2003;41(12):1489-96. https://doi.org/10. 1016/j.brat.2003.07.010.

24. Chew NWS, Lee GKH, Tan BYQ. A multinational, multicentre study on the psychological outcomes and associated physical symptoms amongst healthcare workers during Covid-19 outbreak. Brain Behav Immun. 2020. https://doi.org/10.1016/j.bbi.2020.04.049.

25. Wu KK, Chan KS. The development of the Chinese version of Impact of Event Scale-Revised (CIES-R). Soc Psychiatry Psychiatr Epidemiol. 2003;38(2):94-8. https://doi.org/10.1007/s00127-003-0611-x.

26. Kroenke K, Spitzer RL, Williams JB. The PHQ-9: validity of a brief depression severity measure. J Gen Intern Med. 2001;16(9):606-13.

27. Moriarty AS, Gilbody S, McMillan D, Manea L. Screening and case finding for major depressive disorder using the Patient Health Questionnaire (PHQ-9): a meta-analysis. Gen Hosp Psychiatry. 2015;37(6):567-76. https:// doi.org/10.1016/j.genhosppsych.2015.06.012.

28. Gilbody S, Richards D, Brealey S, Hewitt C. Screening for depression in medical settings with the Patient Health Questionnaire (PHQ): a diagnostic meta-analysis. J Gen Intern Med. 2007;22(11):1596-602. https://doi. org/10.1007/s11606-007-0333-y.

29. Spitzer RL, Kroenke K, Williams JB, Löwe B. A brief measure for assessing generalized anxiety disorder: the GAD-7. Arch Intern Med. 2006;166(10):1092-7. https://doi.org/10.1001/archinte.166.10.1092.

30. Tong X, An D, McGonigal A, Park SP, Zhou D. Validation of the generalized anxiety disorder-7 (GAD-7) among Chinese people with epilepsy. Epilepsy Res. 2016;120:31-6. https://doi.org/10.1016/j.eplepsyres.2015.11. 019.

31. Lee $Y-C$, Lin $Y-C$, Huang C-L, Fredrickson B. The construct and measurement of peace of mind. J Happiness Stud. 2013;14:571-90. https://doi. org/10.1007/s10902-012-9343-5.

32. Sikka P, Pesonen $H$, Revonsuo A. Peace of mind and anxiety in the waking state are related to the affective content of dreams. Sci Rep. 2018;8(1):12762. https://doi.org/10.1038/s41598-018-30721-1.

33. Chalder T, Berelowitz G, Pawlikowska T, Watts L, Wessely S, Wright D, et al. Development of a fatigue scale. J Psychosom Res. 1993;37(2):147-53. https://doi.org/10.1016/0022-3999(93)90081-P.

34. Wong WS, Fielding R. Construct validity of the Chinese version of the Chalder Fatigue Scale in a Chinese community sample. J Psychosom Res. 2010;68(1):89-93.

35. Buysse DJ, Reynolds CF 3rd, Monk TH, Berman SR, Kupfer DJ. The Pittsburgh Sleep Quality Index: a new instrument for psychiatric practice and research. Psychiatry Res. 1989;28(2):193-213. https://doi.org/10.1016/ 0165-1781(89)90047-4.

36. Tsai PS, Wang SY, Wang MY, Su CT, Yang TT, Huang CJ, et al. Psychometric evaluation of the Chinese version of the Pittsburgh Sleep Quality Index (CPSQI) in primary insomnia and control subjects. Qual Life Res. 2005;14(8):1943-52. https://doi.org/10.1007/s11136-005-4346-x.

37. Baer RA, Smith GT, Hopkins J, Krietemeyer J, Toney L. Using self-report assessment methods to explore facets of mindfulness. Assessment. 2006;13(1):27-45. https://doi.org/10.1177/1073191105283504.

38. Hou J, Wong SY, Lo HH, MakWW, Ma HS. Validation of a Chinese version of the Five Facet Mindfulness Questionnaire in Hong Kong and development of a short form. Assessment. 2014;21(3):363-71. https://doi.org/10. 1177/1073191113485121.
39. Sahdra B, Ciarrochi J, Parker P. Nonattachment and mindfulness: related but distinct constructs. Psychol Assess. 2016;28(7):819-29. https://doi. org/10.1037/pas0000264.

40. Sahdra BK, Shaver PR, Brown KW. A scale to measure nonattachment: a Buddhist complement to Western research on attachment and adaptive functioning. J Pers Assess. 2010;92(2):116-27. https://doi.org/10.1080/ 00223890903425960 (PMID: 20155561).

41. Sahdra BK, Ciarrochi J, Parker PD, Marshall S, Heaven P. Empathy and nonattachment independently predict peer nominations of prosocial behavior of adolescents. Front Psychol. 2015;19(6):263. https://doi.org/10. 3389/fpsyg.2015.00263.

42. Mak WWS, Ng ISW, Wong CCY, Law RW. Resilience Style Questionnaire: development and validation among college students and cardiac patients in Hong Kong. Assessment. 2019;26(4):706-25. https://doi.org/ 10.1177/1073191116683798.

43. Li J, Mo PK, Wu AM, Lau JT. Roles of self-stigma, social support, and positive and negative affects as determinants of depressive symptoms among HIV infected men who have sex with men in China. AIDS Behav. 2017;21(1):261-73. https://doi.org/10.1007/s10461-016-1321-1.

44. Righy C, Rosa RG, da Silva RTA, Kochhann R, Migliavaca CB, Robinson CC, et al. Prevalence of post-traumatic stress disorder symptoms in adult critical care survivors: a systematic review and meta-analysis. Crit Care. 2019;23(1):213. https://doi.org/10.1186/s13054-019-2489-3.

45. Kim SH, Schneider SM, Bevans M, Kravitz L, Mermier C, Qualls C, et al. PTSD symptom reduction with mindfulness-based stretching and deep breathing exercise: randomized controlled clinical trial of efficacy. J Clin Endocrinol Metab. 2013;98(7):2984-92. https://doi.org/10.1210/jc. 2012-3742.

46. Mak WW, Chio FH, Chan AT, Lui WW, Wu EK. The efficacy of internet-based mindfulness training and cognitive-behavioral training with telephone support in the enhancement of mental health among college students and young working adults: randomized controlled trial. J Med Internet Res. 2017;19(3):e84. https://doi.org/10.2196/jmir.6737.

47. Guo L, Wu M, Zhu Z, Zhang L, Peng S, Li W, et al. Effectiveness and influencing factors of online education for caregivers of patients with eating disorders during COVID-19 pandemic in China. Eur Eat Disord Rev. 2020. https://doi.org/10.1002/erv.2783.10.1002/erv.2783.

48. Querstret D, Cropley M, Fife-Schaw C. Internet-based instructor-led mindfulness for work-related rumination, fatigue, and sleep: assessing facets of mindfulness as mechanisms of change. A randomized waitlist control trial. J Occup Health Psychol. 2017;22(2):153-69. https://doi.org/10.1037/ ocp0000028.

49. Tyler Boden M, Bernstein A, Walser RD, Bui L, Alvarez J, Bonn-Miller MO. Changes in facets of mindfulness and posttraumatic stress disorder treatment outcome. Psychiatry Res. 2012;200(2-3):609-13. https://doi.org/10. 1016/j.psychres.2012.07.011.

50. Husain SF, Yu R, Tang TB, Tam WW, Tran B, Quek TT, et al. Validating a functional near-infrared spectroscopy diagnostic paradigm for Major Depressive Disorder. Sci Rep. 2020;10(1):9740. https://doi.org/10.1038/ s41598-020-66784-2.

51. Ho CSH, Lim LJH, Lim AQ, Chan NHC, Tan RS, Lee SH, et al. Diagnostic and predictive applications of functional near-infrared spectroscopy for major depressive disorder: a systematic review. Front Psychiatry. 2020;6(11):378. https://doi.org/10.3389/fpsyt.2020.00378.

Ready to submit your research? Choose BMC and benefit from:

- fast, convenient online submission

- thorough peer review by experienced researchers in your field

- rapid publication on acceptance

- support for research data, including large and complex data types

- gold Open Access which fosters wider collaboration and increased citations

- maximum visibility for your research: over 100M website views per year

At BMC, research is always in progress.

Learn more biomedcentral.com/submissions 